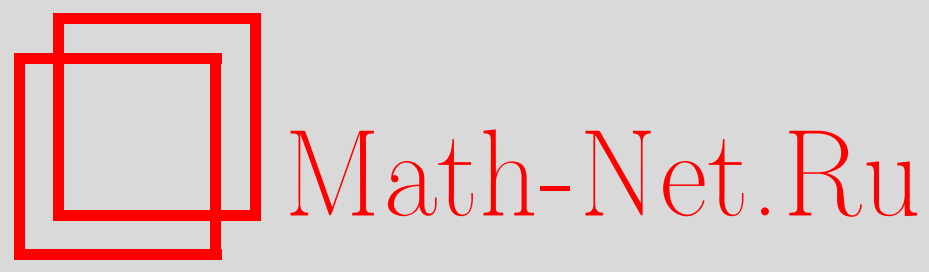

В. М. Бухштабер, Д. В. Лейкин, В. З. Энольский, Рекурсивное семейство дифференциальных полиномов, порожденное тождеством Сильвестра, и теоремы сложения для гиперэллиптических функций Клейна, Функи. анализ и его прил., 1997, том 31, выпуск 4, 19-33

DOI: https://doi.org/10.4213/faa489

Использование Общероссийского математического портала Math$\mathrm{Net.Ru}$ подразумевает, что вы прочитали и согласны с пользовательским соглашением

http://www . mathnet.ru/rus/agreement

Параметры загрузки:

IP : 34.227 .88 .159

26 апреля 2023 г., 16:49:54

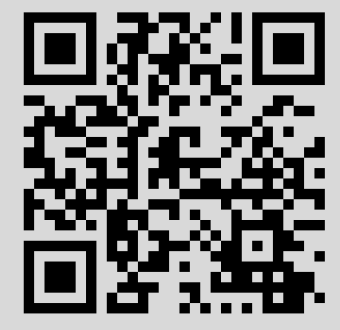




\title{
Рекурсивное семейство дифференциальных полиномов, порожденное тождеством Сильвестра, и теоремы сложения для гиперэллиптических функций Клейна
}

\author{
(c) 1997. В. М. БухштАБеР, Д. В. ЛЕйкин, В. З. ЭнольСКий
}

В. И. Арнольду к его шестидесятилетию

\section{Введение}

В настоящей работе вводится рекурсивное семейство функций $\left\{F_{n}\right\}_{n=1,2, \ldots}$, порожденное соотношениями

$$
F_{n+1} F_{n-1}=\lambda\left(F_{n} \partial_{x y} F_{n}-\partial_{x} F_{n} \partial_{y} F_{n}\right)+\mu f(x, y) F_{n}^{2}
$$

с начальными условиями $F_{0}=1, F_{1}=f(x, y)$. Показано, что $F_{n}(x, y)$ для любого $n$ является целочисленным полиномом относительно функции $f(x, y)$, ее производных и параметров $\lambda, \mu$. Эти дифференциальные полиномы $F_{n}(x, y)=$ $D_{n}(f(x, y))=D_{n}(f(x, y) ; \lambda, \mu)$ представляют собой главные миноры одной матрицы, а само семейство $\left\{D_{n}(f)\right\}$ порождается тождеством Сильвестра для составных определителей.

Мы применяем эту рекурсию к классической проблеме обобщения на случай гиперэллиптических $\sigma$-функций Клейна (род $g>1$ ) соотношения для эллиптических функций Вейерштрасса

$$
\frac{\sigma(u+v) \sigma(u-v)}{\sigma(u)^{2} \sigma(v)^{2}}=\wp(v)-\wp(u),
$$

играющего ключевую роль в теории и приложениях эллиптических функций (род $g=1)$.

Гиперэллиптическая $\sigma$-Функция рода $g$ определяется как элемент кольца римановых $\theta$-функций, автоморфный относительно действия модулярной группы $\operatorname{Sp}(2 g, \mathbb{Z})$. Логарифмические производные $\sigma$-функции

$$
\wp_{i j}=-\frac{\partial^{2}}{\partial u_{i} \partial u_{j}} \ln \sigma(\boldsymbol{u}), \quad \wp_{i, j, k}=-\frac{\partial^{3}}{\partial u_{i} \partial u_{j} \partial u_{k}} \ln \sigma(\boldsymbol{u}), \quad i, j, k=1, \ldots, g,
$$

и т.д. являются гиперэллиптическими абелевыми функциями, т.е. $2 g$-периодическими мероморфными функциями.

В случае рода $g=1$ поле эллиптических функций порождается эллиптическими функциями Вейерштрасса $\wp, \wp^{\prime}$, униформизуюшими эллиптическую кривую. В случае $g>1$ гиперэллиптические функции $\wp_{i j}, \wp_{i j k}$ униформизуют

* Эта работа была частично финансирована грантом CRDF UM1-325 и грантом РФФИ № 96-01-01404. 
якобиан $\operatorname{Jac}(V)$ гиперэллиптической кривой $V$, причем четные функции $\wp_{i j}$ униформизуют многообразие $\operatorname{Kуммера~} \operatorname{Kum}(V)=\operatorname{Jac}(V) /(\boldsymbol{u} \rightarrow-\boldsymbol{u})$, определяемое как фактор якобиана по гиперэллиптической инволюции.

Следующий результат является одним из основных в этой работе.

Для гиперэллиптической $\sigma$-функции Клейна рода $g \geqslant 1$ имеет место соотношение

$$
\frac{\sigma(\boldsymbol{u}+\boldsymbol{v}) \sigma(\boldsymbol{u}-\boldsymbol{v})}{\sigma(\boldsymbol{u})^{2} \sigma(\boldsymbol{v})^{2}}=D_{g}\left(\wp_{g g}(\boldsymbol{v})-\wp_{g g}(\boldsymbol{u}) ; \frac{1}{4}, \frac{1}{2}\right),
$$

где $\wp_{g g}(\boldsymbol{v})-\wp_{g g}(\boldsymbol{u})$ рассматривается как функция от $x=u_{g}+v_{g}$ и $y=u_{g}-v_{g}$, a $D_{g}(;)$ - дифференциальный полином, задаваемый рекурсией (0.1).

Бейкер [1] доказал, исходя из формулы Кёнигсбергера [2]

$$
\sigma(\boldsymbol{u}+\boldsymbol{v}) \sigma(\boldsymbol{u}-\boldsymbol{v})=\sum_{k=1}^{2^{g}} \sigma\left[\varepsilon_{R}-\varepsilon_{i}\right](\boldsymbol{u})^{2} \sigma\left[\varepsilon_{i}\right](\boldsymbol{v})^{2},
$$

что в случае, когда $\sigma$ - гиперэллиптическая функция Клейна, имеет место соотношение

$$
\frac{\sigma(\boldsymbol{u}+\boldsymbol{v}) \sigma(\boldsymbol{u}-\boldsymbol{v})}{\sigma(\boldsymbol{u})^{2} \sigma(\boldsymbol{v})^{2}}=\mathscr{F}_{g}(\boldsymbol{u}, \boldsymbol{v})
$$

где $\mathscr{F}_{g}(\boldsymbol{u}, \boldsymbol{v})$ представляет собой полином от $\wp_{i j}(\boldsymbol{u})$ и $\wp_{i j}(\boldsymbol{v})$. Здесь $\varepsilon_{R}-$ характеристика вектора римановых констант, а характеристики $\varepsilon_{i}$ принадлежат подгруппе $A_{2^{g}}$ группы полуцелых характеристик, порожденной $g$ сдвигами на полупериоды, соответствующие наперед выбранным $g$ точкам ветвления рассматриваемой гиперэллиптической кривой. Бейкер описал алгоритм построения таких полиномов, основанный на перевыражении отношений квадратов $\sigma[\varepsilon]$-функций через функции $\wp_{i, j}$, и вычислил эти полиномы для родов 2 и 3. В случае старших родов этот алгоритм остается неэффективным даже при современных средствах аналитических вычислений. Применение рекурсивного семейства (0.1) позволило эффективно решить эту задачу. А именно, используя полученное нами описание соотношений между функциями $\wp_{i, j}$ и их производными $($ см. $[4,5])$, мы выражаем дифференциальный полином $D_{g}\left(\wp_{g g}(\boldsymbol{v})-\wp_{g g}(\boldsymbol{u}) ; \frac{1}{4}, \frac{1}{2}\right)$ $=\mathscr{F}_{g}(\boldsymbol{u}, \boldsymbol{v})$ в виде пфаффиана кососимметрической матрицы, матричные элементы которой явно заданы как квадратичные формы от векторов $\left\{\wp_{i j}(\boldsymbol{u})\right\}$ и $\left\{\wp_{i j}(\boldsymbol{v})\right\}$.

В $\S 1$ статьи описывается рекурсивное семейство дифференциальных полиномов безотносительно гиперэллиптических теорем сложения. Мы надеемся, что это семейство имеет более широкую область применения.

В $\S 2$ описывается универсальное расслоение главнополяризованных абелевых многообразий как мероморфный образ произведения $\mathbb{C}^{g}$ и расширенного полупространства Зигеля, осуществляемый функщиями, инвариантными относительно действия группы сдвигов на периоды и модулярной группы $\operatorname{Sp}(2 g, \mathbb{Z})$. Эта конструкция, суженная на случай гиперэллиптических кривых, приводит к определению гиперэллиптических функций Клейна. В этом же параграфе приведена реализация гиперэллиптических многообразий Якоби и Куммера как алгебраических подмногообразий [3-5], являющихся пересечением кубик и квартик соответственно. 
В $\S 3$ результаты первых двух параграфов объединяются для получения сформулированных выше основных результатов.

Завершая введение, подчеркнем, что предложенные теоремы сложения являются алгебраическими. Кроме того, эти теоремы носят универсальный характер, т. е., используя уравнения ограничения, с помощью формул, приведенных во втором разделе, можно сужать набор базисных функций вплоть до явного определения минимального базиса из $g+1$ абелевых функций (в котором произвольная функция абелева мероморфного поля выражается рационально), существование которого утверждается теоремами Зигеля [6] и Бохнера [7].

Мы предполагаем что наши теоремы сложения являются характеристическими, т. е. характеризуют гиперэллиптические абелевы функщии в том же смысле, в котором известные теоремы Эрмита и Вейерштрасса характеризуют класс эллиптических функций (см., например, [8]).

Приложения обсуждаемых теорем сложения к двумерному уравнению Шрёдингера и уравнению Кортевега де Фриза на решетке даны в $[9,10]$.

\section{§1. Рекурсивное семейство дифференциальных полиномов, порожденное тождеством Сильвестра для определителей}

ТЕорема 1.1. Для произвольной $C^{\infty}$-функиии $f(x, y)$ и произвольньх комплексных чисел $\lambda$ и рекурсия, заданная соотношением

$$
F_{n+1} F_{n-1}=\lambda\left(F_{n} \partial_{x y} F_{n}-\partial_{x} F_{n} \partial_{y} F_{n}\right)+\mu f(x, y) F_{n}^{2}
$$

с начальными условиями $F_{0}=1$ u $F_{1}=f(x, y)$, определяет $\left\{F_{n}\right\}_{n=1,2, \ldots}$ как семейство полиномов с иельми коэффициентами относительно функиии $f(x, y)$, ее производных и параметров $\lambda, \mu$.

Эти полиномь можно представить в виде определителей $F_{n}=\operatorname{det} M_{n}$, где $M_{n}=\left\{m_{i, j}\right\}_{i, j=1, \ldots, n}$, причем $m_{1,1}=f(x, y), m_{1, k}=\eta \partial_{x} m_{1, k-1}, k>1$, $m_{i, 1}=\xi \partial_{y} m_{i-1,1}, i>1$, п прочие матричнье элементь удовлетворяют соотношениям

$$
\begin{aligned}
& m_{i, k}=\eta \partial_{x} m_{i, k-1}+\sum_{j=1}^{i-1} C_{j}^{i-1} \mu m_{i-j, 1} m_{j, k-1}, \quad k>1, i>1, \\
& m_{i, k}=\xi \partial_{y} m_{i-1, k}+\sum_{j=1}^{k-1} C_{j}^{k-1} \mu m_{1, k-j} m_{i-1, j}, \quad i>1, k>1,
\end{aligned}
$$

әде $C_{l}^{k}=k ! /(l !(k-l) !)$ - биномиальнье коэффициентьи и $\eta \xi=\lambda$.

ДокАЗАтЕльство. Прежде всего покажем, что матрицы $M_{k}$ существуют для произвольной функции $f(x, y)$, т. е. что соотношения $(\mathfrak{x})$ и $(\mathfrak{y})$ совместны. Доказательство проведем по индукции. Действительно,

$$
M_{2}=\left(\begin{array}{cc}
f(x, y) & \eta \partial_{x} f(x, y) \\
\xi \partial_{y} f(x, y) & \lambda \partial_{x y} f(x, y)+\mu f(x, y)^{2}
\end{array}\right)
$$

и совместность $(\mathfrak{x})$ и $(\mathfrak{y})$ очевидна при $i \leqslant 2$ и $k \leqslant 2$. Предположим, что совместность $(\mathfrak{x})$ и $(\mathfrak{y})$ имеет место для всех $m_{j, l}$ при $j=1, \ldots, i-1$ и $l=1, \ldots, k-1$, 
и проверим, что выражения, которые получаются из $m_{i, k}$ последовательным перевыражением с помощью $(\mathfrak{x})$ и $(\mathfrak{y})$ и, в обратном порядке, $(\mathfrak{y})$ и $(\mathfrak{x})$ совпадают. Получаем соответственно

$$
\begin{aligned}
& \lambda \partial_{x y} m_{i-1, k-1}-\sum_{l=1}^{k-2} \sum_{j=1}^{i-2} C_{l}^{k-2} C_{j}^{i-2} \mu^{2} m_{j, l} m_{i-j-1,1} m_{1, k-l-1} \\
& \quad+\sum_{l=1}^{k-2} C_{l}^{k-2} \mu\left(m_{1, k-l} m_{i-1, l}+m_{1, k-l-1} m_{i-1, l+1}\right)+\sum_{j=1}^{i-1} C_{j}^{i-1} \mu m_{i-j, 1} m_{j, k-1} \\
& \lambda \partial_{x y} m_{i-1, k-1}-\sum_{l=1}^{k-2} \sum_{j=1}^{i-2} C_{l}^{k-2} C_{j}^{i-2} \mu^{2} m_{j, l} m_{i-j-1,1} m_{1, k-l-1} \\
& \quad+\sum_{j=1}^{i-2} C_{j}^{i-2} \mu\left(m_{i-j, 1} m_{j, k-1}+m_{j-i-1,1} m_{j+1, k-1}\right)+\sum_{l=1}^{k-1} C_{l}^{k-1} \mu m_{1, k-l} m_{i-1, l} .
\end{aligned}
$$

Разность последних выражений обращается в 0, поскольку биномиальные коэффициенты удовлетворяют тождеству $C_{l}^{k}-C_{l}^{k-1}-C_{l-1}^{k-1} \equiv 0$. Индукция завершена.

Покажем, что $\operatorname{det} M_{n-1}, \operatorname{det} M_{n}$ и $\operatorname{det} M_{n+1}$ удовлетворяют соотношению (1.1).

Лемма 1.2. Для любых $n \times n$-матрии $A$ u $B$ имеет место тождество

$\operatorname{det}\left(B \boldsymbol{a}_{1}, \boldsymbol{a}_{2}, \ldots, \boldsymbol{a}_{n}\right)+\operatorname{det}\left(\boldsymbol{a}_{1}, B \boldsymbol{a}_{2}, \ldots, \boldsymbol{a}_{n}\right)+\cdots+\operatorname{det}\left(\boldsymbol{a}_{1}, \boldsymbol{a}_{2}, \ldots, B \boldsymbol{a}_{n}\right)$

$$
=(\operatorname{det} A)(\operatorname{tr} B) \text {, }
$$

где $\boldsymbol{a}_{k}$ есть $k$-й столбеи матриць $A$.

ДокАЗАтЕльство леммы. Раскрывая определители в левой части (1.2), получаем, что коэффициент при матричном элементе $B_{i, k}$ равен определителю матрицы, которая получается из матрицы $A$ заменой $i$-й строки на строку с номером $k$, т. е. равен $\delta_{i, k} \operatorname{det} A$.

Используя $(\mathfrak{x})$ и $(1.2)$, вычислим $\eta \partial_{x} \operatorname{det} M_{n}$. В силу $(\mathfrak{x})$ имеем

$$
\eta \partial_{x} \boldsymbol{m}_{j}=\boldsymbol{m}_{j+1}-\mathscr{B}_{n} \boldsymbol{m}_{j}
$$

где $\boldsymbol{m}_{j}=\left(m_{1, j}, \ldots, m_{n, j}\right)^{T}$ и матрица $\mathscr{B}_{n}=\left\{C_{k}^{l-1} \mu m_{l-k, 1}\right\}_{l, k=1, \ldots, n}$ (полагаем $m_{l, 1} \equiv 0$ при $\left.l \leqslant 0\right)$, очевидно, имеет нулевой след. Далее получаем

$$
\begin{aligned}
\eta \partial_{x} \operatorname{det} M_{n} & =\operatorname{det}\left(\eta \partial_{x} \boldsymbol{m}_{1}, \boldsymbol{m}_{2}, \ldots, \boldsymbol{m}_{n}\right)+\cdots+\operatorname{det}\left(\boldsymbol{m}_{1}, \boldsymbol{m}_{2}, \ldots, \eta \partial_{x} \boldsymbol{m}_{n}\right) \\
& =\operatorname{det}\left(\boldsymbol{m}_{2}-\mathscr{B}_{n} \boldsymbol{m}_{1}, \ldots, \boldsymbol{m}_{n}\right)+\cdots+\operatorname{det}\left(\boldsymbol{m}_{1}, \ldots, \boldsymbol{m}_{n+1}-\mathscr{B}_{n} \boldsymbol{m}_{n}\right) \\
& =\operatorname{det}\left(\boldsymbol{m}_{1}, \ldots, \boldsymbol{m}_{n-1}, \boldsymbol{m}_{n+1}\right)
\end{aligned}
$$

где мы использовали, что $\mathscr{B}_{n}$ имеет нулевой след. Аналогично, из (y) следует, что

$$
\xi \partial_{y} \operatorname{det} M_{n}=\operatorname{det}\left(\widehat{\boldsymbol{m}}_{1}, \ldots, \widehat{\boldsymbol{m}}_{n}\right),
$$

где мы положили $\widehat{\boldsymbol{m}}_{j}=\left(m_{1, j}, \ldots, m_{n-1, j}, m_{n+1, j}\right)^{T}$. В силу $(\mathfrak{x})$ имеем

$$
\eta \partial_{x} \widehat{\boldsymbol{m}}_{j}=\widehat{\boldsymbol{m}}_{j+1}-\widehat{\mathscr{B}}_{n} \widehat{\boldsymbol{m}}_{j}-\mu m_{1,1} m_{n, j} \boldsymbol{e}_{n},
$$


где $\widehat{\mathscr{B}}_{n}$ получается из матрицы $\mathscr{B}_{n+1}$ вычеркиванием $n$-х строки и столбца, а $\boldsymbol{e}_{n}=(0, \ldots, 0,1)^{T}$. След матрицы $\widehat{\mathscr{B}}_{n}$ также равен 0 . Тогда

$$
\begin{aligned}
\eta \partial_{x}\left(\xi \partial_{y} \operatorname{det} M_{n}\right)= & \operatorname{det}\left(\eta \partial_{x} \widehat{\boldsymbol{m}}_{1}, \widehat{\boldsymbol{m}}_{2}, \ldots, \widehat{\boldsymbol{m}}_{n}\right)+\cdots+\operatorname{det}\left(\widehat{\boldsymbol{m}}_{1}, \widehat{\boldsymbol{m}}_{2}, \ldots, \eta \partial_{x} \widehat{\boldsymbol{m}}_{n}\right) \\
= & \operatorname{det}\left(\widehat{\boldsymbol{m}}_{2}-\widehat{\mathscr{B}}_{n} \widehat{\boldsymbol{m}}_{1}-\mu m_{1,1} m_{n, 1} \boldsymbol{e}_{n}, \ldots, \widehat{\boldsymbol{m}}_{n}\right)+\cdots \\
& \quad+\operatorname{det}\left(\widehat{\boldsymbol{m}}_{1}, \ldots, \widehat{\boldsymbol{m}}_{n+1}-\widehat{\mathscr{B}}_{n} \boldsymbol{m}_{n}-\mu m_{1,1} m_{n, n} \boldsymbol{e}_{n}\right) \\
= & \operatorname{det}\left(\widehat{\boldsymbol{m}}_{1}, \ldots, \widehat{\boldsymbol{m}}_{n-1}, \widehat{\boldsymbol{m}}_{n+1}\right)-\mu m_{1,1} \operatorname{det} M_{n} .
\end{aligned}
$$

Заметим, что матрицы $\left(\boldsymbol{m}_{1}, \ldots, \boldsymbol{m}_{n-1}, \boldsymbol{m}_{n+1}\right),\left(\widehat{\boldsymbol{m}}_{1}, \ldots, \widehat{\boldsymbol{m}}_{n}\right)$ и $\left(\widehat{\boldsymbol{m}}_{1}, \ldots\right.$, $\left.\widehat{\boldsymbol{m}}_{n-1}, \widehat{\boldsymbol{m}}_{n+1}\right)$ суть подматрицы матрицы $M_{n+1}$, получаемые вычеркиванием $(n+1)$-й строки и $n$-го столбца, $n$-й строки и $(n+1)$-го столбца и $n$-й строки и $n$-го столбца соответственно. Мы можем применить тождество Сильвестра:

$$
\operatorname{det} M_{n+1} \operatorname{det} M_{n-1}=\operatorname{det}\left(\begin{array}{cc}
\operatorname{det} M_{n} & \operatorname{det}\left(\boldsymbol{m}_{1}, \ldots, \boldsymbol{m}_{n-1}, \boldsymbol{m}_{n+1}\right) \\
\operatorname{det}\left(\widehat{\boldsymbol{m}}_{1}, \ldots, \widehat{\boldsymbol{m}}_{n}\right) & \operatorname{det}\left(\widehat{\boldsymbol{m}}_{1}, \ldots, \widehat{\boldsymbol{m}}_{n-1}, \widehat{\boldsymbol{m}}_{n+1}\right)
\end{array}\right) \text {, }
$$

которое, используя $(1.3),(1.4)$ и (1.5), перепишем в виде

$$
\operatorname{det} M_{n+1} \operatorname{det} M_{n-1}=\operatorname{det}\left(\begin{array}{cc}
\operatorname{det} M_{n} & \eta \partial_{x} \operatorname{det} M_{n} \\
\xi \partial_{y} \operatorname{det} M_{n} & \left(\lambda \partial_{x y}+\mu m_{1,1}\right) \operatorname{det} M_{n}
\end{array}\right) .
$$

Таким образом, учитывая, что по определению $m_{1,1}=f(x, y)$, мы доказали, что для $F_{n-1}=\operatorname{det} M_{n-1}, F_{n}=\operatorname{det} M_{n}$ и $F_{n+1}=\operatorname{det} M_{n+1}$ соотношение (1.1) выполняется при всех $n=1,2, \ldots$.

ЗАмечАния. 1. Тождество (1.2) является частным случаем тождества $\operatorname{tr}_{n+1}\left(B, A_{1}, \ldots, A_{n}\right)=0$, верного для любого набора $n \times n$-матрищ $B, A_{1}, \ldots$, $A_{n}$, где $\operatorname{tr}_{k+1}\left(B, A_{1}, \ldots, A_{k}\right)$ есть $(k+1)$-след $((k+1)$-характер Фробениуса), определяемый по формуле [14]

$$
\operatorname{tr}_{k+1}\left(B, A_{1}, \ldots, A_{k}\right)=(\operatorname{tr} B) \operatorname{tr}_{k}\left(A_{1}, \ldots, A_{k}\right)-\sum_{i=1}^{k} \operatorname{tr}_{k}\left(A_{1}, \ldots, B A_{i}, \ldots, A_{k}\right) \text {. }
$$

Здесь $\operatorname{tr} B$ - обычный след матрицы $B$. В случае когда $A_{i}$ является $n \times n$-матрицей, у которой $j$-й столбец равен $a_{j} \delta_{i j}$, где $a_{j}$ - это $j$-й столбец матрицы $A$, то $\operatorname{tr}_{n}\left(A_{1}, \ldots, A_{n}\right)=\operatorname{det} A$.

2. В обозначениях теоремы 1.1 положим

$$
G=G(x, y ; u, v)=1+\sum_{u \geqslant 1, v \geqslant 1} m_{i j}(x, y) \frac{u^{i}}{i !} \frac{v^{j}}{j !} .
$$

Тогда функщия $G$ однозначно определяется как решение (в формальных рядах по $u$ и $v$ ) системы уравнений

$$
\begin{aligned}
\left(\partial_{v}-\eta \partial_{x}\right) \partial_{u} G & =f(x, y+\xi u) G, \\
\partial_{v}\left(\partial_{u}-\xi \partial_{y}\right) G & =f(x+\eta v, y) G
\end{aligned}
$$

с начальными условиями $G(x, y ; 0, v)=G(x, y ; u, 0)=1$. Сушествование матриц $M_{k}, k>1$, эквивалентно совместности этих уравнений, которая очевидна.

3. Непосредственная проверка показывает, что, положив $F_{-m}=\mu^{2 m-1} F_{m-1}$ для $m \geqslant 1$, мы получаем функции $F_{n}(x, y)$, удовлетворяющие соотношениям (1.1) для всех целых $n$. 


\section{§2. Гиперэллиптические функции Клейна}

2.1. Универсальное расслоение абелевых многообразий. Пусть $\mathbb{H}^{g}$ - некоторое $g$-мерное линейное пространство над телом кватернионов $\mathbb{H}$. Рассмотрим подгруппы $\operatorname{Sp}(2 g, \mathbb{Z})$ и $\mathbb{Z}^{g} \times \mathbb{Z}^{g}$ группы движений пространства $\mathbb{H}^{g}$. Фиксируя изоморфизм $\mathbb{H}^{g} \cong \mathbb{C}^{g} \times \mathbb{C}^{g}$, будем записывать векторы из $\mathbb{H}^{g}$ в виде векторов-строк $\boldsymbol{v}=\left(\boldsymbol{v}_{1}, \boldsymbol{v}_{2}\right)$, где $\boldsymbol{v}_{1}, \boldsymbol{v}_{2} \in \mathbb{C}^{g}$. Для $t \in \operatorname{Sp}(2 g, \mathbb{Z})$, т. е. такого $t$, что

$$
\operatorname{det} t=1 \quad \text { и } \quad t\left(\begin{array}{cc}
0 & -1_{g} \\
1_{g} & 0
\end{array}\right) t^{T}=\left(\begin{array}{cc}
0 & -1_{g} \\
1_{g} & 0
\end{array}\right),
$$

положим $t=\left(\begin{array}{ll}a^{T} & b^{T} \\ c^{T} & d^{T}\end{array}\right)$, где $a, b, c, d \in \mathrm{GL}(g, \mathbb{Z})$. Зафиксируем правое действие группы $\operatorname{Sp}(2 g, \mathbb{Z})$ на $\mathbb{H}^{g}$ следующим образом:

$$
\boldsymbol{v} \cdot g=\left(\boldsymbol{v}_{1} d+\boldsymbol{v}_{2} c, \boldsymbol{v}_{1} b+\boldsymbol{v}_{2} a\right) .
$$

Правое действие группы трансляций $\mathbb{Z}^{g} \times \mathbb{Z}^{g}$ будем записывать в виде

$$
\boldsymbol{v} \cdot\left(\begin{array}{c}
\boldsymbol{m} \\
\boldsymbol{m}^{\prime}
\end{array}\right)=\left(\boldsymbol{v}_{1}+\boldsymbol{m}^{T}, \boldsymbol{v}_{2}+\boldsymbol{m}^{\prime T}\right) .
$$

Обозначим через $A_{\mathbb{H}}(g, \mathbb{Z})$ подгруппу группы движений пространства $\mathbb{H}^{g}$, порожденную подгруппами $\operatorname{Sp}(2 g, \mathbb{Z})$ и $\mathbb{Z}^{g} \times \mathbb{Z}^{g}$. Эта группа $A_{\mathbb{H}}(g, \mathbb{Z})$ является целочисленным аналогом аффинной группы и представляет собой расширение группы $\operatorname{Sp}(2 g, \mathbb{Z})$ с помощью $\mathbb{Z}^{g} \times \mathbb{Z}^{g}$, т. е. имеет место точная последовательность

$$
0 \rightarrow \mathbb{Z}^{g} \times \mathbb{Z}^{g} \stackrel{i}{\rightarrow} A_{\mathbb{H}}(g, \mathbb{Z}) \stackrel{\pi}{\rightarrow} \mathrm{Sp}(2 g, \mathbb{Z}) \rightarrow 0,
$$

где $i$ - каноническое вложение, а проекция $\pi$ переводит аффинное преобразование $\gamma \in A_{\mathbb{H}}(g, \mathbb{Z})$ в соответствующее вращение $\pi(\gamma) \in \operatorname{Sp}(2 g, \mathbb{Z})$, т.е.

$$
\boldsymbol{v} \cdot \pi(\gamma)=\boldsymbol{v} \cdot \gamma-\mathbf{0} \cdot \gamma \cdot
$$

Это расширение соответствует следующему действию группы $\operatorname{Sp}(2 g, \mathbb{Z})$ автоморфизмами группы $\mathbb{Z}^{g} \times \mathbb{Z}^{g}$ :

$$
\left(\begin{array}{c}
\boldsymbol{m} \\
\boldsymbol{m}^{\prime}
\end{array}\right) \cdot g=\left(\begin{array}{c}
\widehat{\boldsymbol{m}} \\
\widehat{\boldsymbol{m}}^{\prime}
\end{array}\right)=\left(\begin{array}{c}
d^{T} \boldsymbol{m}+c^{T} \boldsymbol{m}^{\prime} \\
b^{T} \boldsymbol{m}+a^{T} \boldsymbol{m}^{\prime}
\end{array}\right) .
$$

Напомним понятие верхнего полупространства Зигеля. Это пространство матриц $\mathscr{S}_{g}=\left\{\tau \in \mathrm{GL}(g, \mathbb{C}) \mid \tau^{T}=\tau, \operatorname{Im}(\tau)\right.$ положительно определена $\}$. Введем расширение пространства $\mathscr{S}_{g}$, которое обозначим через $\mathscr{S}_{g}^{\prime}$, как пространство $g \times 2 g$-матриц $\left(2 \omega, 2 \omega^{\prime}\right)$, таких, что их столбцы линейно независимы над $\mathbb{R}$, матрицы $\omega$ и $\omega^{\prime}$ удовлетворяют уравнению

$$
\omega^{\prime} \omega^{T}-\omega \omega^{\prime T}=0
$$

$\operatorname{det}(\omega) \neq 0$ и $\tau=\omega^{-1} \omega^{\prime} \in \mathscr{S}_{g}$. Такие матрицы $\left(2 \omega, 2 \omega^{\prime}\right) \in \mathscr{S}_{g}^{\prime}$ порождают так называемые главнополяризованные решетки в $\mathbb{C}^{g}$. Имеется естественная 
проекция на верхнее полупространство Зигеля $\pi_{s}: \mathscr{S}_{g}^{\prime} \rightarrow \mathscr{S}_{g},\left(\omega, \omega^{\prime}\right) \mapsto \omega^{-1} \omega^{\prime}$, и вложение $i_{s}: \mathscr{S}_{g} \rightarrow \mathscr{S}_{g}^{\prime}, \tau \mapsto\left(1_{g}, \tau\right)$.

Действие (2.1) индуцирует правое действие группы $\mathrm{Sp}(2 g, \mathbb{Z})$ на $\mathscr{S}_{g}^{\prime}$ :

$$
\left(\omega, \omega^{\prime}\right) \cdot t=\left(\widehat{\omega}, \widehat{\omega}^{\prime}\right)=\left(\omega d+\omega^{\prime} c, \omega b+\omega^{\prime} a\right),
$$

которое переводится проекцией $\pi_{s}: \mathscr{S}_{g}^{\prime} \rightarrow \mathscr{S}_{g}$ в каноническое правое действие группы $\operatorname{Sp}(2 g, \mathbb{Z})$ на верхнем полупространстве Зигеля:

$$
\pi_{s}\left(\left(\omega, \omega^{\prime}\right) \cdot t\right)=\pi_{s}\left(\widehat{\omega}, \widehat{\omega}^{\prime}\right)=\left(\omega d+\omega^{\prime} c\right)^{-1}\left(\omega b+\omega^{\prime} a\right)=(d+\tau c)^{-1}(b+\tau a) .
$$

Таким образом, каноническое действие группы $\operatorname{Sp}(2 g, \mathbb{Z})$ на $\mathscr{S}_{g}$ разлагается в композицию $\tau \cdot g=\pi_{s}\left(i_{s}(\tau) \cdot g\right)$.

Рассмотрим пространства $\mathscr{U}_{g}=\mathbb{C}^{g} \times \mathscr{S}_{g}$ и $\mathscr{U}_{g}^{\prime}=\mathbb{C}^{g} \times \mathscr{S}_{g}^{\prime}$. Действие $(2.5)$ группы $\operatorname{Sp}(2 g, \mathbb{Z})$ на $\mathscr{S}_{g}^{\prime}$ продолжается до правого действия группы $A_{\mathbb{H}}(g, \mathbb{Z})$ на $\mathscr{U}_{g}^{\prime}$ следующим образом.

Пусть $\left(\boldsymbol{z}, \omega, \omega^{\prime}\right) \in \mathscr{U}_{g}^{\prime}$ и $\gamma=\left(t,\left(\begin{array}{c}\boldsymbol{m} \\ \boldsymbol{m}^{\prime}\end{array}\right)\right) \in A_{\mathbb{H}}(n, \mathbb{Z}) ;$ тогда

$$
\left(\boldsymbol{z}, \omega, \omega^{\prime}\right) \cdot \gamma=\left(\boldsymbol{z}+2 \widehat{\omega} \boldsymbol{m}+2 \widehat{\omega}^{\prime} \boldsymbol{m}^{\prime}, \widehat{\omega}, \widehat{\omega}^{\prime}\right),
$$

т. е. подгруппа $\mathbb{Z}^{g} \times \mathbb{Z}^{g}$ действует на $\mathscr{U}_{g}^{\prime}$ преобразованиями $\left(1_{2 g},\left(\begin{array}{c}\boldsymbol{m} \\ \boldsymbol{m}^{\prime}\end{array}\right)\right)$ :

$$
\left(\boldsymbol{z}, \omega, \omega^{\prime}\right) \mapsto\left(\boldsymbol{z}+2 \omega \boldsymbol{m}+2 \omega^{\prime} \boldsymbol{m}^{\prime}, \omega, \omega^{\prime}\right),
$$

а модулярная подгруппа $\operatorname{Sp}(2 g, \mathbb{Z})-$ как $\left(t,\left(\begin{array}{l}\mathbf{0} \\ \mathbf{0}\end{array}\right)\right)$ :

$$
\left(\boldsymbol{z}, \omega, \omega^{\prime}\right) \mapsto\left(\boldsymbol{z}, \widehat{\omega}, \widehat{\omega}^{\prime}\right)=\left(\boldsymbol{z}, \omega d+\omega^{\prime} c, \omega b+\omega^{\prime} a\right) .
$$

Проекция $\pi_{s}: \mathscr{S}_{g}^{\prime} \rightarrow \mathscr{S}_{g}$ продолжается до проекции $\tilde{\pi}_{s}: \mathscr{U}_{g}^{\prime} \rightarrow \mathscr{U}_{g}$ по формуле $\tilde{\pi}_{s}\left(\boldsymbol{z}, \omega, \omega^{\prime}\right)=\left(\omega^{-1} \boldsymbol{z}, \omega^{-1} \omega^{\prime}\right)$, а вложение $i_{s}: \mathscr{S}_{g} \rightarrow \mathscr{S}_{g}^{\prime}$ продолжается до вложения $\tilde{\imath}_{s}: \mathscr{U}_{g} \rightarrow \mathscr{U}_{g}^{\prime}$ согласно формуле $\tilde{\imath}_{s}(\boldsymbol{z}, \tau)=(\boldsymbol{z}, 1, \tau)$. Ясно, что $\tilde{\pi}_{s} \tilde{\imath}_{s}=\mathrm{id}$.

ОПРЕДЕЛЕНИЕ 1. Факторпространство $\mathscr{U} \mathscr{T}_{g}^{\prime}=\mathscr{U}_{g}^{\prime} / A_{\mathbb{H}}(g, \mathbb{Z})$ называется универсальным пространством g-мерных главнополяризованных абелевых многообразий.

Каноническая проекция $\mathscr{U}_{g}^{\prime} \rightarrow \mathscr{S}_{g}^{\prime}$ индуцирует проекцию факторпространств $p: \mathscr{U}_{g}^{\prime} \rightarrow \mathscr{U}_{\mathscr{M}}^{\prime}$. Тройка $\left(\mathscr{U}_{g}^{\prime}, \mathscr{U}_{\mathscr{M}}^{\prime}, p\right)$ называется универсальныл расслоением g-мерных главнополяризованных абелевых многообразий.

По построению слой $p^{-1}(m)$ над точкой $m \in \mathscr{U} \mathscr{M}_{g}^{\prime}$ является $g$-мерным главнополяризованным абелевым многообразием, которое есть фактор пространства $\mathbb{C}^{g}$ по решетке, порожденной столбцами матрицы $\left(\omega, \omega^{\prime}\right)$, принадлежащей классу эквивалентности матриц $m$.

2.2. $\boldsymbol{\sigma}$-функции. Для произвольной точки $\left(\boldsymbol{z}, \omega, \omega^{\prime}\right)$ определим характеpuстику при помощи отображения char: $\mathscr{U}_{g}^{\prime} \rightarrow \mathbb{R}^{g} \times \mathbb{R}^{g},\left(\boldsymbol{z}, \omega, \omega^{\prime}\right) \mapsto[\varepsilon]$, согласно уравнению $\boldsymbol{z}=2\left(\omega, \omega^{\prime}\right)[\varepsilon]$, которое единственным образом определяет $[\varepsilon]$ благодаря линейной независимости столбцов матрицы $\left(\omega, \omega^{\prime}\right)$ над $\mathbb{R}$.

Определим $\theta[\varepsilon]$-функиию с характеристикой на $\mathscr{U}_{g}^{\prime}$ как функцию $\theta[\varepsilon]$ : $\mathscr{U}_{g}^{\prime} \rightarrow \mathbb{C}$, заданную сходящимся рядом Фурье

$\theta[\varepsilon]\left(\boldsymbol{z}, \omega, \omega^{\prime}\right)=\sum_{\boldsymbol{m} \in \mathbb{Z}^{g}} \exp \pi i\left\{\left(\boldsymbol{m}+\boldsymbol{\varepsilon}^{\prime}\right)^{T} \omega^{-1} \omega^{\prime}\left(\boldsymbol{m}+\boldsymbol{\varepsilon}^{\prime}\right)+\left(\omega^{-1} \boldsymbol{z}+2 \boldsymbol{\varepsilon}\right)^{T}\left(\boldsymbol{m}+\boldsymbol{\varepsilon}^{\prime}\right)\right\}$ 
для произвольного вектора $[\varepsilon]=\left(\varepsilon, \varepsilon^{\prime}\right)^{T} \in \mathbb{R}^{g} \times \mathbb{R}^{g}$, который называется характеристикой $\theta$-функции.

Под действием трансляций (2.7) $\theta[\varepsilon]$-функция преобразуется следуюшим обра30м:

$$
\begin{aligned}
& \theta[\varepsilon]\left(\boldsymbol{z}+2 \omega \boldsymbol{m}+2 \omega^{\prime} \boldsymbol{m}^{\prime}, \omega, \omega^{\prime}\right) \\
& \quad=\exp \left\{-\pi i\left[\boldsymbol{m}^{\prime T}\left(\omega^{-1} \boldsymbol{z}+\boldsymbol{m}^{\prime}\right)-\boldsymbol{m}^{T} \boldsymbol{\varepsilon}^{\prime}+\boldsymbol{m}^{\prime T} \varepsilon\right]\right\} \theta[\varepsilon]\left(\boldsymbol{z}, \omega, \omega^{\prime}\right) .
\end{aligned}
$$

При преобразованиях $(2.8)$ из $\operatorname{Sp}(2 g, \mathbb{Z})$ функция $\theta[\varepsilon]$ переходит в $\theta[\hat{\varepsilon}]$, причем преобразованная характеристика имеет вид

$$
[\hat{\varepsilon}]=\left(\begin{array}{cc}
d^{T} & -c^{T} \\
-b^{T} & a^{T}
\end{array}\right)[\varepsilon]+\frac{1}{2}\left[\begin{array}{c}
-\operatorname{diag}\left(c^{T} d\right) \\
\operatorname{diag}\left(a^{T} b\right)
\end{array}\right]
$$

полностью преобразование имеет вид

$$
\theta[\hat{\varepsilon}]\left(\boldsymbol{z}, \widehat{\omega}, \widehat{\omega}^{\prime}\right)=\left(\operatorname{det}\left(\omega^{-1} \widehat{\omega}\right)\right)^{1 / 2} \exp \left\{\frac{1}{4} \pi i \boldsymbol{z}^{T}\left(\omega^{T}\right)^{-1} c \widehat{\omega}^{-1} \boldsymbol{z}\right\} \theta[\varepsilon]\left(\boldsymbol{z}, \omega, \omega^{\prime}\right) .
$$

Построим модификацию функции $\theta[\varepsilon]$, которая преобразуется наиболее простым образом под действием (2.8). Для этого нам понадобятся так называемые ассочиированнье матричь.

Матрица $\left(\eta, \eta^{\prime}\right)$, удовлетворяющая для данной матрищы $\left(\omega, \omega^{\prime}\right) \in \mathscr{S}_{g}^{\prime}$ условию

$$
\left(\begin{array}{cc}
\omega & \omega^{\prime} \\
\eta & \eta^{\prime}
\end{array}\right)\left(\begin{array}{cc}
0 & -1_{g} \\
1_{g} & 0
\end{array}\right)\left(\begin{array}{cc}
\omega & \omega^{\prime} \\
\eta & \eta^{\prime}
\end{array}\right)^{T}=-\frac{1}{2} \pi i\left(\begin{array}{cc}
0 & -1_{g} \\
1_{g} & 0
\end{array}\right),
$$

называется ассоциированной $c\left(\omega, \omega^{\prime}\right)$. Ассоциированная матрица не единственна. Действительно, (2.13) эквивалентно уравнениям

$$
\omega^{\prime} \omega^{T}-\omega \omega^{\prime T}=0, \quad \eta^{\prime} \omega^{T}-\eta \omega^{\prime T}=-\frac{\pi i}{2} 1_{g}, \quad \eta^{\prime} \eta^{T}-\eta \eta^{\prime T}=0,
$$

первое из которых выполняется в силу (2.4). Чтобы решить остальные, возьмем произвольную матрицу $\varkappa$ и положим $\eta(\varkappa)=2 \varkappa \omega$ и $\eta^{\prime}(\varkappa)=2 \varkappa \omega^{\prime}-\frac{1}{2} \pi i\left(\omega^{T}\right)^{-1}$; тогда прочие уравнения также сводятся к (2.4). Таким образом, для любых $\varkappa_{1}$ и $\varkappa_{2}$ обе матрицы $\left(\eta\left(\varkappa_{1}\right), \eta^{\prime}\left(\varkappa_{1}\right)\right)$ и $\left(\eta\left(\varkappa_{2}\right), \eta^{\prime}\left(\varkappa_{2}\right)\right)$ ассоциированны с $\left(\omega, \omega^{\prime}\right)$.

Под действием (2.8) ассоциированная матрица переходит в ассоциированную, т. е. $\left(\hat{\eta}, \hat{\eta}^{\prime}\right)$ и $\left(\widehat{\omega}, \widehat{\omega}^{\prime}\right)$ удовлетворяют $(2.13)$, где $\left(\eta, \eta^{\prime}\right)$ преобразуется по правилу $\left(\hat{\eta}, \hat{\eta}^{\prime}\right)=\left(\eta d+\eta^{\prime} c, \eta b+\eta^{\prime} a\right)$.

ЛЕмма 2.1. Пусть $\left(\eta, \eta^{\prime}\right)$ - произвольная матрича, ассочиированная c $\left(\omega, \omega^{\prime}\right)$. Тогда матрича $\varkappa=\frac{1}{2} \eta \omega^{-1}$ под действием преобразований из группь $\operatorname{Sp}(2 g, \mathbb{Z})$ преобразуется по правилу $\widehat{\varkappa}=\varkappa-\frac{1}{4} \pi i\left(\omega^{T}\right)^{-1} c \widehat{\omega}^{-1}$.

ДокаЗАТЕльСтво. Учитывая соотношение $\eta^{\prime}=2 \varkappa \omega^{\prime}-\frac{1}{2} \pi i\left(\omega^{T}\right)^{-1}$, имеем

$$
\begin{aligned}
\widehat{\varkappa} & =\frac{1}{2} \hat{\eta}(\widehat{\omega})^{-1}=\frac{1}{2}\left(\eta d+\eta^{\prime} c\right)(\widehat{\omega})^{-1}=\left(\varkappa \omega d+\varkappa \omega^{\prime} c-\frac{\pi i}{4}\left(\omega^{T}\right)^{-1} c\right)(\widehat{\omega})^{-1} \\
& =\varkappa\left(\omega d+\omega^{\prime} c\right)(\widehat{\omega})^{-1}-\frac{\pi i}{4}\left(\omega^{T}\right)^{-1} c(\widehat{\omega})^{-1}=\varkappa-\frac{\pi i}{4}\left(\omega^{T}\right)^{-1} c(\widehat{\omega})^{-1} .
\end{aligned}
$$

Теперь мы можем дать 
ОПРЕДЕЛЕНИЕ 2. Фиксируя некоторую матрицу $\left(\eta, \eta^{\prime}\right)$, ассоциированную с $\left(\omega, \omega^{\prime}\right) \in \mathscr{S}_{g}^{\prime}$, определим $\sigma[\varepsilon]$-функиию с характеристикой как функцию $\sigma[\varepsilon]: \mathscr{U}_{g}^{\prime} \rightarrow \mathbb{C}$, заданную формулой

$$
\sigma[\varepsilon]\left(\boldsymbol{z}, \omega, \omega^{\prime}\right)=\frac{C\left(\omega, \omega^{\prime}\right)}{\sqrt{\operatorname{det}(\omega)}} \exp \left\{\frac{1}{2} \boldsymbol{z}^{T} \eta \omega^{-1} \boldsymbol{z}\right\} \theta[\varepsilon]\left(\boldsymbol{z}, \omega, \omega^{\prime}\right),
$$

где $C\left(\omega, \omega^{\prime}\right)$ - некоторый инвариант действия группы $\operatorname{Sp}(2 g, \mathbb{Z})$.

Из (2.10) и (2.12) следует

Теорема 2.2. Функиия $\sigma[\varepsilon]$ обладает следуюшими трансформачионнылми свойствами:

1. Трансляии: положим $\boldsymbol{E}\left(\boldsymbol{m}, \boldsymbol{m}^{\prime}\right)=\eta \boldsymbol{m}+\eta^{\prime} \boldsymbol{m}^{\prime} u \boldsymbol{\Omega}\left(\boldsymbol{m}, \boldsymbol{m}^{\prime}\right)=\omega \boldsymbol{m}+$ $\omega^{\prime} \boldsymbol{m}^{\prime}$, где $\boldsymbol{m}, \boldsymbol{m}^{\prime} \in \mathbb{Z}^{g} ;$ тогда

$$
\begin{aligned}
\sigma[\varepsilon]\left(\boldsymbol{z}+2 \boldsymbol{\Omega}\left(\boldsymbol{m}, \boldsymbol{m}^{\prime}\right), \omega, \omega^{\prime}\right)=\exp \left\{2 \boldsymbol{E}^{T}\left(\boldsymbol{m}, \boldsymbol{m}^{\prime}\right)\left(\boldsymbol{z}+\boldsymbol{\Omega}\left(\boldsymbol{m}, \boldsymbol{m}^{\prime}\right)\right)\right\} \\
\times \exp \left\{-\pi i \boldsymbol{m}^{T} \boldsymbol{m}^{\prime}-2 \pi i \varepsilon^{T} \boldsymbol{m}^{\prime}\right\} \sigma[\varepsilon]\left(\boldsymbol{z}, \omega, \omega^{\prime}\right) .
\end{aligned}
$$

2. Модулярнье преобразования:

$$
\sigma[\hat{\varepsilon}]\left(\boldsymbol{z}, \widehat{\omega}, \widehat{\omega}^{\prime}\right)=\sigma[\varepsilon]\left(\boldsymbol{z}, \omega, \omega^{\prime}\right),
$$

причем $[\varepsilon]$ преобразуется $\kappa[\hat{\varepsilon}]$ согласно (2.11).

2.3. $\sigma$-функции на гиперэллиптических якобианах. Рассмотрим частный случай, когда матрицы $\left(2 \omega, 2 \omega^{\prime}\right)$ возникают как матрицы периодов голоморфных дифференциалов на римановой поверхности $V$ гиперэллиптической кривой $V(x, y)$ рода $g$, заданной формулой $V(x, y)=\left\{(x, y) \in \mathbb{C}^{2} \mid f(x, y)=0\right\}$, где $f(x, y)$ - полином

$$
y^{2}-\sum_{i=0}^{2 g+2} \lambda_{i} x^{i}=y^{2}-\lambda_{2 g+2} \prod_{k=1}^{2 g+2}\left(x-e_{k}\right) .
$$

Зададим канонический базис в 1 -мерной группе когомологий $H^{1}(V, \mathbb{C})$ в виде

$$
d \boldsymbol{u}^{T}=\left(d u_{1}, \ldots, d u_{g}\right), \quad d u_{i}=\frac{x^{i-1} d x}{y} .
$$

Пусть $(\mathfrak{a}, \mathfrak{b})$ - базис циклов в $H_{1}(V, \mathbb{Z})$ с симплектической матрицей пересечений. Каноническое спаривание $H^{1}(V, \mathbb{C}) \times H_{1}(V, \mathbb{Z}) \rightarrow \mathbb{C}$ определяет матрицу $\left(\left\{\oint_{\mathfrak{a}_{i}} d u_{j}\right\},\left\{\oint_{\mathfrak{b}_{i}} d u_{j}\right\}\right)_{i, j=1, \ldots, g}=\left(2 \omega, 2 \omega^{\prime}\right)$ и, таким образом, приводит к специальному подпространству пространства $\mathscr{S}_{g}^{\prime}$, которое мы будем обозначать через $\mathscr{H}_{g}^{\prime}$.

Ограничим приведенную выше конструкцию на подпространство $\mathscr{U}_{g}^{\prime}=$ $\mathbb{C}^{g} \times \mathscr{H}_{g}^{\prime} \subset \mathscr{U}_{g}^{\prime}$. Подпространство $\mathscr{H}_{g}^{\prime} \subset \mathscr{S}_{g}^{\prime}$ замкнуто относительно действия группы $A_{\mathbb{H}}(g, \mathbb{Z})$.

ОПРЕДЕЛЕНИЕ 3. Факторпространство $\mathscr{H}_{\mathscr{M}}^{\prime}=\mathscr{H}_{g}^{\prime} / A_{\mathbb{H}}(g, \mathbb{Z})$ называется пространством модулей якобианов римановых поверхностей гиперэллиптических кривых рода $g$. Расслоение $p_{\mathscr{H}}: \mathscr{H}_{g}^{\prime} \rightarrow \mathscr{H}_{\mathscr{M}}^{\prime}$, индуцированное вложением $\mathscr{H} \mathscr{M}_{g}^{\prime} \subset \mathscr{U} \mathscr{M}_{g}^{\prime}$, называется универсальным расслоением якобианов римановых поверхностей гиперэллиптических кривых рода g. Пространство $\mathscr{H} \mathscr{T}_{g}^{\prime}$ 
этого расслоения называется универсальным пространством якобианов римановых поверхностей гиперэллиптических кривых рода $g$.

На $V$ для канонического базиса голоморфных дифференциалов имеется ассоциированный базис мероморфных дифференциалов, а именно, дифференциалы

$$
d \boldsymbol{r}^{T}=\left(d r_{1}, \ldots, d r_{g}\right), \quad \text { где } d r_{j}=\sum_{k=j}^{2 g+1-j}(k+1-j) \lambda_{k+1+j} \frac{x^{k} d x}{4 y},
$$

имеют матрицу периодов $\left(2 \eta, 2 \eta^{\prime}\right)=\left(\left\{-\oint_{\mathfrak{a}_{i}} d r_{j}\right\},\left\{-\oint_{\mathfrak{b}_{i}} d r_{j}\right\}\right)_{i, j=1, \ldots, g}$, которая только множителем 2 отличается от матрицы $\left(\eta, \eta^{\prime}\right)$, ассоциированной с $\left(\omega, \omega^{\prime}\right)$.

Примем во внимание то обстоятельство, что в $\mathscr{U} \mathscr{H}_{g}^{\prime}$ имеется выделенный класс точек вида $\left(\boldsymbol{K}_{R}, \omega, \omega^{\prime}\right)$, переходящих под действием $(2.8)$ в $\left(\widehat{\boldsymbol{K}}_{R}, \widehat{\omega}, \widehat{\omega}^{\prime}\right)$. Вектор $\boldsymbol{K}_{R}=\sum_{k=1}^{g+1} \int_{\infty}^{e_{2 k}} d \boldsymbol{u}$ - это вектор римановых констант. Суммируя изложенное, приходим к такому определению:

ОПРЕДЕлЕНИЕ 4. Фундаментальная гиперэллиптическая б-функиия это функция $\sigma: \mathscr{U}_{g}^{\prime} \rightarrow \mathbb{C}$, заданная формулой

$$
\sigma\left(\boldsymbol{z}, \omega, \omega^{\prime}\right)=\frac{1}{\sqrt[4]{D(V)}} \sqrt{\frac{\pi^{g}}{\operatorname{det}(\omega)}} \exp \left\{\frac{1}{2} \boldsymbol{z}^{T} \eta \omega^{-1} \boldsymbol{z}\right\} \theta\left[\varepsilon_{R}\right]\left(\boldsymbol{z}, \omega, \omega^{\prime}\right),
$$

где $\left[\varepsilon_{R}\right]$ - характеристика вектора римановых констант, а величина $D(V)-$ дискриминант (по $x$ ) определяющего кривую $V(x, y)$ уравнения $f(x, y)=0$.

Фундаментальная гиперэллиптическая $\sigma$-функция обладает свойством

$$
\sigma\left(\boldsymbol{z}, \omega, \omega^{\prime}\right)=\sigma\left(\boldsymbol{z}, \widehat{\omega}, \widehat{\omega}^{\prime}\right),
$$

и, более того, любая ее вторая логарифмическая производная является функцией, автоморфной относительно действия (2.6). А именно, пусть

$$
\wp_{i j}\left(\boldsymbol{z}, \omega, \omega^{\prime}\right)=-\frac{\partial^{2}}{\partial z_{i} \partial z_{j}} \ln \sigma\left(\boldsymbol{z}, \omega, \omega^{\prime}\right) ;
$$

тогда для любого $\gamma \in A_{\mathbb{H}}(g, \mathbb{Z})$

$$
\wp_{i j}\left(\left(\boldsymbol{z}, \omega, \omega^{\prime}\right) \cdot \gamma\right)=\wp_{i j}\left(\boldsymbol{z}, \omega, \omega^{\prime}\right) .
$$

ПредЛОЖЕНИЕ 2.3. Функции $\wp_{i, k}$, где $i, k=1, \ldots, g$, как функиии от $\left(\boldsymbol{z}, \omega, \omega^{\prime}\right)$ определяют функции на универсальном пространстве гиперэллиптических якобианов $\mathscr{H}_{g}^{\prime}$, а как функчии от $\boldsymbol{z}$ при фиксированной матриче $\left(\omega, \omega^{\prime}\right)$ они являются абелевыми, т. е. $2 g$-периодическими мероморфными функииями на якобиане $\mathrm{Jac}(V)$ римановой поверхности соответствующей гиперэллиттической кривой $V(x, y)$.

Введенная выше функция $\sigma$ и ее логарифмические производные

$$
\zeta_{i}(\boldsymbol{u})=\frac{\partial \ln \sigma(\boldsymbol{u})}{\partial u_{i}}, \quad \wp_{i j}(\boldsymbol{u})=-\frac{\partial^{2} \ln \sigma(\boldsymbol{u})}{\partial u_{i} \partial u_{j}}, \quad \wp_{i j k}(\boldsymbol{u})=-\frac{\partial^{3} \ln \sigma(\boldsymbol{u})}{\partial u_{i} \partial u_{i} \partial u_{k}}, \quad \ldots,
$$


где $i, j, k, \ldots=1, \ldots, g$, рассматриваемые как функции на гиперэллиптическом якобиане $\operatorname{Jac}(V)$, называются гиперэллиптическими $\sigma-, \zeta-u$ ю-функциями Клейна. Приведем их важнейшие свойства.

Аналитическое разложение в нуле целой функции $\sigma$ имеет вид

$$
\sigma(\boldsymbol{u})=\operatorname{det}\left(\left\{u_{i+j-1}\right\}_{i, j=1, \ldots,[(g+1) / 2]}\right)+\text { слагаемые старших порядков. }
$$

Введем, следуя [11], функции

$$
\begin{aligned}
h_{i k}= & 4 \wp_{i-1, k-1}-2 \wp_{k, i-2}-2 \wp_{i, k-2} \\
& +\frac{1}{2}\left(\delta_{i k}\left(\lambda_{2 i-2}+\lambda_{2 k-2}\right)+\delta_{k, i+1} \lambda_{2 i-1}+\delta_{i, k+1} \lambda_{2 k-1}\right),
\end{aligned}
$$

где $i, k=1, \ldots, g+2$, предполагая при этом, что $\wp_{n m}=0$, если $n<1$ или $m<1$, а также что $\wp_{n m}=0$, если $n>g$ или $m>g$. Очевидно, что $h_{i j}=h_{j i}$. Обозначим матрицу $\left(h_{i k}\right)$ через $H$.

Теорема 2.4. Ранг матриць $H$ не превосходит 3 , что эквивалентно выполнению соотношений

$$
\operatorname{det} H\left[\begin{array}{l}
i, j, g+1, g+2 \\
k, l, g+1, g+2
\end{array}\right]=0
$$

для любых $i, j, k, l=1, \ldots, g$. Кроме того, для любых $i, k=1, \ldots, g$ выполняются соотношения

$$
\wp_{g g i} \wp_{g g k}=-\frac{1}{4} \operatorname{det} H\left[\begin{array}{l}
i, g+1, g+2 \\
k, g+1, g+2
\end{array}\right] .
$$

В общей точке якобиана $\mathrm{Jac}(V)$ ранг матрицы $H$ равен 3 , в полупериодах он равен 2, в общей точке б-дивизора $(\sigma)$ ранг матрицьл $\sigma^{2}(\boldsymbol{u}) H$ равен 3 и в точках $(\sigma)_{\operatorname{sing}}=\left\{\boldsymbol{u} \mid \sigma(\boldsymbol{u})=0, \partial \sigma(\boldsymbol{u}) / \partial u_{k}=0 \forall k=1, \ldots, g\right\}$ он равен 0 .

Здесь $H\left[\begin{array}{l}i_{1}, \ldots, i_{k} \\ j_{1}, \ldots, j_{k}\end{array}\right]$ обозначает $k \times k$-матрицу, составленную из строк с номерами $i_{1}, \ldots, i_{k}$ и столбцов с номерами $j_{1}, \ldots, j_{k}$ матрицы $H$.

Пересечение $g(g+1) / 2$ кубик (2.18) определяет гиперэллиптическое многообразие Якоби $\mathrm{Jac}(V) \backslash(\sigma)$ как алгебраическую поверхность в $\mathbb{C}^{g+\frac{1}{2} g(g+1)}$. Пересечение $g(g-1) / 2$ квартик (2.17) определяет гиперэллиптическое многообразие Куммера $\operatorname{Jac}(V) \backslash(\sigma) / \pm$ как алгебраическую поверхность в $\mathbb{C}^{\frac{1}{2}} g(g+1)$.

Таким образом, поле мероморфных функций на $\mathrm{Jac}(V)$ реализуется системой функций с алгебраическими связями. Эта система замкнута по отношению к операции дифференцирования; в частности,

$$
\wp_{g g g i}=\left(6 \wp_{g g}+\lambda_{2 g}\right) \wp_{g i}+6 \wp_{g, i-1}-2 \wp_{g-1, i}+\frac{1}{2} \delta_{g i} \lambda_{2 g-1} \cdot
$$

Подробно функции Клейна и их приложения обсуждаются в работе авторов [5].

\section{§3. Теоремы сложения для гиперэллиптических функций Клейна}

3.1. Обозначения. Пусть род кривой $V(x, y)$ равен $g$. Введем функции $m_{i, k}: \operatorname{Jac}(V) \times \operatorname{Jac}(V) \rightarrow \mathbb{C}$ по формуле

$$
\begin{aligned}
m_{i, k}(\boldsymbol{u}, \boldsymbol{v})= & \wp_{g, i+1}(\boldsymbol{u}) \wp_{g, k+1}(\boldsymbol{v})-\wp_{g, i+1}(\boldsymbol{v}) \wp_{g, k+1}(\boldsymbol{u}) \\
& +\wp_{i, k+1}(\boldsymbol{v})-\wp_{i, k+1}(\boldsymbol{u})-\wp_{i+1, k}(\boldsymbol{v})+\wp_{i+1, k}(\boldsymbol{u}),
\end{aligned}
$$


где $\boldsymbol{v}, \boldsymbol{u} \in \operatorname{Jac}(V)$ - произвольные точки; очевидно, что $m_{i, k}(\boldsymbol{u}, \boldsymbol{v})$ - четные по $\boldsymbol{u}$ и $\boldsymbol{v}$ функции и

$$
m_{i, k}(\boldsymbol{u}, \boldsymbol{v})=-m_{k, i}(\boldsymbol{u}, \boldsymbol{v})=m_{k, i}(\boldsymbol{v}, \boldsymbol{u})=-m_{i, k}(\boldsymbol{v}, \boldsymbol{u}) .
$$

Заметим, что $m_{i, k}(\boldsymbol{u}, \boldsymbol{v})$ зависит от рода $g$.

Далее, пусть $k \in \mathbb{N}$. Построим кососимметрическую $2\left[\frac{k+1}{2}\right] \times 2\left[\frac{k+1}{2}\right]$-матрицу

$$
\mathscr{M}_{k}(\boldsymbol{u}, \boldsymbol{v})=\left\{m_{i, j}(\boldsymbol{u}, \boldsymbol{v})\right\}, \quad i, j \in\left\{g-k, \ldots, g-\frac{1}{2}\left(1+(-1)^{k}\right)\right\} ;
$$

таким образом, при $k=2 n+1$ и $k=2 n+2$ имеем $(2 n+2) \times(2 n+2)$-матрицы.

Обозначим пфаффиан матрицы $\mathscr{M}_{k}(\boldsymbol{u}, \boldsymbol{v})$ через $\mathscr{F}_{k}(\boldsymbol{u}, \boldsymbol{v})$ :

$$
\mathscr{F}_{k}(\boldsymbol{u}, \boldsymbol{v})=\operatorname{pf} \mathscr{M}_{k}(\boldsymbol{u}, \boldsymbol{v})=\sqrt{\operatorname{det} \mathscr{M}_{k}(\boldsymbol{u}, \boldsymbol{v})},
$$

причем знак при извлечении квадратного корня выбирается так, чтобы

$$
\text { pf }\left(\begin{array}{cc}
0 & -1_{n} \\
1_{n} & 0
\end{array}\right)=(-1)^{n+1} .
$$

Непосредственная проверка показывает, что $m_{i, j}(\boldsymbol{u}, \boldsymbol{u})=0$ и $\left(\partial m_{i, j} / \partial u_{q}\right)(\boldsymbol{u}, \boldsymbol{u})$ $=0$ для всех $i, j$ и $q$. Следовательно, $\mathscr{F}_{k}(\boldsymbol{u}, \boldsymbol{u})=0$ и $\left(\partial \mathscr{F}_{k} / \partial u_{q}\right)(\boldsymbol{u}, \boldsymbol{u})=0$ для всех $k$ и $q$.

3.2. Основная формула и примеры. В $[12,11,3-5]$ нами развита теория гиперэллиптических функций Клейна. Это позволило получить следующий результат:

ТЕОРема 3.1. Пфаффиань $\mathscr{F}_{k}(\boldsymbol{u}, \boldsymbol{v}), k \geqslant 1$, матрицы $\mathscr{M}_{k}(\boldsymbol{u}, \boldsymbol{v})$ удовлетворяют рекурсивному соотношению

$$
4 \mathscr{F}_{k+1}(\boldsymbol{u}, \boldsymbol{v}) \mathscr{F}_{k-1}(\boldsymbol{u}, \boldsymbol{v})=\left[\left(\frac{\partial^{2}}{\partial u_{g}^{2}}-\frac{\partial^{2}}{\partial v_{g}^{2}}\right) \ln \mathscr{F}_{k}(\boldsymbol{u}, \boldsymbol{v})+2 \mathscr{F}_{1}(\boldsymbol{u}, \boldsymbol{v})\right] \mathscr{F}_{k}^{2}(\boldsymbol{u}, \boldsymbol{v})
$$

для любого рода $g$ при начальном условии $\mathscr{F}_{0}=1$.

Применяя теорему 1.1 и используя то, что по построению $\mathscr{F}_{1}(\boldsymbol{u}, \boldsymbol{v})=m_{g-1, g}$ $=\wp_{g g}(\boldsymbol{v})-\wp_{g g}(\boldsymbol{u})$, а $\mathscr{F}_{k}(\boldsymbol{u}, \boldsymbol{v}) \equiv 0$ при $k>g$, получаем

СлЕДСТвИЕ 3.2. 1. Для любого рода $g \geqslant 1$ имеет место формула

$$
\mathscr{F}_{k}(\boldsymbol{u}, \boldsymbol{v})=D_{k}\left(\wp_{g g}(\boldsymbol{v})-\wp_{g g}(\boldsymbol{u}) ; \frac{1}{4}, \frac{1}{2}\right), \quad k \geqslant 1,
$$

где $\wp_{g g}(\boldsymbol{v})-\wp_{g g}(\boldsymbol{u})$ рассматривается как функиия от $x=u_{g}+v_{g} \quad u=$ $u_{g}-v_{g}$, a $D_{k}($,$) -$ (1.1)

2. Функция $\mathscr{F}_{g}(\boldsymbol{u}, \boldsymbol{v})$ удовлетворяет дифференциальному уравнению

$$
\left(\frac{\partial^{2}}{\partial u_{g}^{2}}-\frac{\partial^{2}}{\partial v_{g}^{2}}\right) \ln \mathscr{F}_{g}(\boldsymbol{u}, \boldsymbol{v})+2\left(\wp_{g g}(\boldsymbol{v})-\wp_{g g}(\boldsymbol{u})\right)=0 .
$$

Теперь все готово для того чтобы получить теорему сложения для гиперэллиптических $\sigma$-функций Клейна: 
ТЕорема 3.3. Для любого рода $g$ имеет место соотношение

$$
\frac{\sigma(\boldsymbol{u}+\boldsymbol{v}) \sigma(\boldsymbol{u}-\boldsymbol{v})}{\sigma(\boldsymbol{u})^{2} \sigma(\boldsymbol{v})^{2}}=\mathscr{F}_{g}(\boldsymbol{u}, \boldsymbol{v}) .
$$

ДокАЗАТЕльСТво. Наш результат вытекает из следуюших фактов. Заметим, что функция

$$
\phi=\log \frac{\sigma(\boldsymbol{u}+\boldsymbol{v}) \sigma(\boldsymbol{u}-\boldsymbol{v})}{\sigma(\boldsymbol{u})^{2} \sigma(\boldsymbol{v})^{2}}
$$

является решением линейного уравнения

$$
\left(\frac{\partial^{2}}{\partial u_{g}^{2}}-\frac{\partial^{2}}{\partial v_{g}^{2}}\right) \phi+2\left(\wp_{g g}(\boldsymbol{v})-\wp_{g g}(\boldsymbol{u})\right)=0 .
$$

Абелева функция

$$
\Phi(\boldsymbol{u}, \boldsymbol{v})=\frac{\sigma(\boldsymbol{u}+\boldsymbol{v}) \sigma(\boldsymbol{u}-\boldsymbol{v})}{\sigma(\boldsymbol{u})^{2} \sigma(\boldsymbol{v})^{2}} \frac{1}{\mathscr{F}_{g}(\boldsymbol{u}, \boldsymbol{v})}
$$

обладает следующими свойствами четности:

$$
\Phi(\boldsymbol{u}, \boldsymbol{v})=\Phi(-\boldsymbol{u}, \boldsymbol{v})=\Phi(\boldsymbol{v}, \boldsymbol{u}),
$$

и, кроме того, является решением уравнения

$$
\left(\frac{\partial^{2}}{\partial u_{g}^{2}}-\frac{\partial^{2}}{\partial v_{g}^{2}}\right) \log \Phi(\boldsymbol{u}, \boldsymbol{v})=0 .
$$

Положим $\boldsymbol{v}=\boldsymbol{u} \pm h \boldsymbol{\alpha}$; тогда

$$
\begin{aligned}
\mathscr{F}_{k}(\boldsymbol{u}, \boldsymbol{v}) & =( \pm h)^{\left[\frac{g+1}{2}\right]} \widehat{\gamma}(\boldsymbol{u}, \boldsymbol{\alpha})+O\left(h^{\left[\frac{g+1}{2}\right]+2}\right), \\
\frac{\sigma(\boldsymbol{u}+\boldsymbol{v}) \sigma(\boldsymbol{u}-\boldsymbol{v})}{\sigma(\boldsymbol{u})^{2} \sigma(\boldsymbol{v})^{2}} & =( \pm h)^{\left[\frac{g+1}{2}\right]} \gamma(\boldsymbol{u}, \boldsymbol{\alpha})+O\left(h^{\left[\frac{g+1}{2}\right]+2}\right)
\end{aligned}
$$

и, следовательно, $\Phi(\boldsymbol{u}, \boldsymbol{v})$ регулярна вблизи диагоналей $h=0$.

Из регулярности и свойств четности вытекает, что $\Phi(\boldsymbol{u}, \boldsymbol{v})$ постоянна на $\boldsymbol{u}= \pm \boldsymbol{v}$. Более подробный анализ разложения вблизи $\boldsymbol{u}=\boldsymbol{v}=0$ показывает, что эта постоянная равна 1 . Теперь осталось использовать тот факт, что функция $\log \Phi(\boldsymbol{u}, \boldsymbol{v})=0$ при $\boldsymbol{u} \rightarrow \pm \boldsymbol{v}$ и в то же время как функция от $u_{g}$ и $v_{g}$ она имеет вид $G_{1}\left(u_{g}+v_{g}\right)+G_{2}\left(u_{g}-v_{g}\right)$. Следовательно, $\Phi(\boldsymbol{u}, \boldsymbol{v}) \equiv 1$.

Рассмотрим примеры в малых родах.

В роде $g=1$ мы получаем хорошо известный результат из теории эллиптических функций [13]

$$
\frac{\sigma(u+v) \sigma(u-v)}{\sigma(u)^{2} \sigma(v)^{2}}=\wp(v)-\wp(u) .
$$

Для родов 2 и 3 воспроизводятся результаты Бейкера [1]. А именно, в роде $g=2$ получаем

$$
\frac{\sigma(\boldsymbol{u}+\boldsymbol{v}) \sigma(\boldsymbol{u}-\boldsymbol{v})}{\sigma(\boldsymbol{u})^{2} \sigma(\boldsymbol{v})^{2}}=\wp_{22}(\boldsymbol{u}) \wp_{21}(\boldsymbol{v})-\wp_{21}(\boldsymbol{u}) \wp_{22}(\boldsymbol{v})-\wp_{11}(\boldsymbol{u})+\wp_{11}(\boldsymbol{v}),
$$


а в роде $g=3$ после некоторых преобразований имеем

$$
\begin{gathered}
\frac{\sigma(\boldsymbol{u}+\boldsymbol{v}) \sigma(\boldsymbol{u}-\boldsymbol{v})}{\sigma(\boldsymbol{u})^{2} \sigma(\boldsymbol{v})^{2}}=\left(\wp_{31}(\boldsymbol{v})-\wp_{31}(\boldsymbol{u})\right)\left(\wp_{22}(\boldsymbol{v})-\wp_{22}(\boldsymbol{u})\right)-\left(\wp_{31}(\boldsymbol{v})-\wp_{31}(\boldsymbol{u})\right)^{2} \\
-\left(\wp_{32}(\boldsymbol{v})-\wp_{32}(\boldsymbol{u})\right)\left(\wp_{21}(\boldsymbol{v})-\wp_{21}(\boldsymbol{u})\right) \\
+\left(\wp_{33}(\boldsymbol{v})-\wp_{33}(\boldsymbol{u})\right)\left(\wp_{11}(\boldsymbol{v})-\wp_{11}(\boldsymbol{u})\right) .
\end{gathered}
$$

Выражение для $g=4$ мы привели в [5].

3.3. О сложении гиперэллиптических ю-функций. Из формул (3.1) и (3.3) непосредственно вытекают теоремы сложения для всех гиперэллиптических функций Клейна произвольных родов. Дело теперь заключается в том, чтобы привести соответствующие формулы к виду, удобному для приложений. Ниже мы кратко опишем один из возможных подходов.

Рассмотрим для некоторого рода $g$ равенство

$$
\frac{\sigma(\boldsymbol{u}+\boldsymbol{v}) \sigma(\boldsymbol{u}-\boldsymbol{v})}{\sigma(\boldsymbol{u})^{2} \sigma(\boldsymbol{v})^{2}}=\mathscr{F}_{g}(\boldsymbol{u}, \boldsymbol{v})
$$

очевидно, что для любого $i \in\{1, \ldots, g\}$ имеет место равенство

$$
\left(\frac{\partial}{\partial u_{i}}+\frac{\partial}{\partial v_{i}}\right) \ln \mathscr{F}_{g}(\boldsymbol{u}, \boldsymbol{v})=2\left(\zeta_{i}(\boldsymbol{u}+\boldsymbol{v})-\zeta_{i}(\boldsymbol{u})-\zeta_{i}(\boldsymbol{v})\right) .
$$

Принимая во внимание, что $\zeta_{i}$ является нечетной функцией, и полагая $\boldsymbol{w}=$ $\boldsymbol{- v}-\boldsymbol{u}$, получаем

$$
\zeta_{i}(\boldsymbol{u})+\zeta_{i}(\boldsymbol{v})+\zeta_{i}(\boldsymbol{w})=-\frac{1}{2}\left(\frac{\partial}{\partial u_{i}}+\frac{\partial}{\partial v_{i}}\right) \ln \mathscr{F}_{g}(\boldsymbol{u}, \boldsymbol{v}) \quad \text { и } \quad \boldsymbol{u}+\boldsymbol{v}+\boldsymbol{w} \equiv 0 .
$$

Отсюда следует

ПРЕДЛОЖЕНИЕ 3.4. Для произвольного $g$ полином $\mathscr{F}_{g}(\cdot, \cdot)$ удовлетворлет системе $2 g$ уравнений

$$
\begin{aligned}
& \mathscr{F}_{g}(\boldsymbol{v}, \boldsymbol{w})\left(\frac{\partial}{\partial u_{i}}+\frac{\partial}{\partial v_{i}}\right) \mathscr{F}_{g}(\boldsymbol{u}, \boldsymbol{v})=\mathscr{F}_{g}(\boldsymbol{u}, \boldsymbol{v})\left(\frac{\partial}{\partial v_{i}}+\frac{\partial}{\partial w_{i}}\right) \mathscr{F}_{g}(\boldsymbol{v}, \boldsymbol{w}), \\
& \mathscr{F}_{g}(\boldsymbol{w}, \boldsymbol{u})\left(\frac{\partial}{\partial u_{i}}+\frac{\partial}{\partial v_{i}}\right) \mathscr{F}_{g}(\boldsymbol{u}, \boldsymbol{v})=\mathscr{F}_{g}(\boldsymbol{u}, \boldsymbol{v})\left(\frac{\partial}{\partial w_{i}}+\frac{\partial}{\partial u_{i}}\right) \mathscr{F}_{g}(\boldsymbol{w}, \boldsymbol{u}),
\end{aligned}
$$

где $i=1, \ldots, g$, тогда и только тогда, когда тройка точек $\boldsymbol{u}, \boldsymbol{v}, \boldsymbol{w} \in$ $\operatorname{Jac}(V)$ удовлетворяет условию $\boldsymbol{u}+\boldsymbol{v}+\boldsymbol{w} \equiv 0$ относительно решетки, порожденной кривой $V$.

Функции $\mathscr{F}_{g}$ и их производные представляют собой полиномы от ю-функций Клейна; поэтому описанные здесь теоремы сложения являются алгебраическими теоремами сложения.

В роде 1 формулы (3.4) - это хорошо известная теорема сложения для эллиптических ю-функций. В этом случае только одно из двух возможных уравнений независимо (из-за дополнительного соотношения $\mathscr{F}_{1}(u, v)+\mathscr{F}_{1}(v, w)+$ $\left.\mathscr{F}_{1}(w, u)=0\right)$, скажем,

$$
\frac{\wp^{\prime}(u)-\wp^{\prime}(v)}{\wp(u)-\wp(v)}=\frac{\wp^{\prime}(w)-\wp^{\prime}(u)}{\wp(w)-\wp(u)},
$$


и выражает факт, что точки $u, v$ и $w$, связанные уравнением $u+v+w \equiv 0$, параметризуют пересечение кубики $\wp^{\prime 2}=4 \wp^{3}+\ldots$ с некоторой прямой.

В старших родах ситуация описывается теоремой Абеля. Точки $\boldsymbol{u}, \boldsymbol{v}$ и $\boldsymbol{w}$ параметризуют некоторые $g$-элементные подмножества из $3 g$ нулей (или, эквивалентно, полюсов) некоторой мероморфной функции на кривой $V$. С другой стороны, мероморфный образ якобиана $\mathrm{Jac}(V)$, который получается с помощью ю-функций Клейна, является пересечением кубик. Теоремы сложения для старших родов также допускают геометрическую интерпретацию. Мы будем обсуждать эти вопросы в других наших публикациях.

\section{ЛИТЕРАТУРА}

1. Baker H. F. On the hyperelliptic sigma functions. Am. J. Math., 20, 301-384 (1898).

2. Königsberger $L$. Ueber die Transformation der Abelshen Functionen erster Ordnung. J. Reine Angew. Math., 64 (27), 17-42 (1865).

3. Бухштабер В. М., Лейкин Д. В., Энольский В. З. Матричная реализация гиперэллиптического многообразия Якоби. УМН, 51, вып. 2, 147-148 (1996).

4. Buchstaber V. M., Enolskii V. Z., Leykin D. V. Hyperelliptic Kleinian functions and applications. In: Solitons Geometry and Topology: On the Crossroad, Advances in Math. Sciences, AMS Translations, Ser. 2, Vol. 179, 1997, pp. 1-34.

5. Buchstaber V. M., Enolskii V. Z., Leykin D. V. Kleinian functions, hyperelliptic Jacobians and applications. In: Reviews in Mathematics and Mathematical Physics, Vol. 10, No. 2, Gordon and Breach, London, 1997, pp. 1-125.

6. Siegel C. L. Meromorphe Funktionen auf kompacten analytishen Mannigfaltigkeinten. Göttingen Nachrichten, 71-77, 1955, Werke, III, 216-222.

7. Bochner $S$. On the addition theorem for multiply periodic functions. Proc. Am. Math. Soc., 3, 99-106 (1952).

8. Голубев B. В. Лекции по аналитической теории дифференциальных уравнений. ГИТТЛ, М.-Л., 1950.

9. Бухитабер В. М., Энольский В. 3. Абелевы блоховские решения двумерного уравнения Шрёдингера. УМН, 50, вып. 1, 191-192 (1995).

10. Nijhoff F. W., Enolskii $V$. Z. Integrable mappings of KdV type and hyperelliptic addition theorems. In: Proceedings of the 2nd International Conference on Symmetries and Integrability of Difference Equations (SIDE II), Canterbery, July 1996, Springer, 1997, pp. 1-12.

11. Лейкин Д. В. К вопросу о кубике Вейерштрасса для гиперэллиптических функций. УМН, 50, вып. 6, 191-192 (1995).

12. Бухштабер B. М., Энольский B. 3. Явное описание гиперэллиптического якобиана на основании $\sigma$-функций Клейна. Функц. анализ и его прил., 30, вып. 1 , 57-60 (1996).

13. Bateman H., Erdelyi A. Higher Transcendental Functions, Vol. 2. McGraw-Hill, New York, 1955.

14. Бухитабер B. M., Рuc E. Г. $k$-характеры Фробениуса и $n$-кольцевые гомоморфизмы. УМН, 52, вып. 2, 159-160 (1997).

Московский государственный университет

Поступило в редакцию им. М. В. Ломоносова

5 сентября 1997 г.

Институт магнетизма НАНУ, Киев 